\title{
An Indonesian Pregnant Woman with Lung Cancer and Liver Metastasis : A Case Report
}

\author{
Vito Filbert Jayalie ${ }^{1}$, Dimas Priantono², Zulkifli Amin ${ }^{2 *}$ \\ ${ }^{1}$ Faculty of Medicine, Universitas Indonesia-Dr. Cipto Mangunkusumo National General Hospital, Jakarta, Indonesia \\ ${ }^{2}$ Department of Internal Medicine, Faculty of Medicine, Universitas Indonesia-Dr. Cipto Mangunkusumo National General \\ Hospital, Jakarta, Indonesia
}

\author{
ARTICLE INFO \\ Article history: \\ Received : 02 April 2018 \\ Reviewed: 20 May 2018 \\ Accepted : 12 July 2018 \\ * Corresponding author: \\ Zulkifli Amin \\ Division of Respirology and Critical \\ Illness, Department of Internal \\ Medicine, Faculty of Medicine \\ Universitas Indonesia, Cipto \\ Mangunkusumo National General \\ Hospital, Jakarta, Indonesia \\ zulkifliamin52@gmail.com
}

\begin{abstract}
A B STR A C T
Background: Lung cancer in pregnancy is a rare disease compared to other types of cancer. However, special issue should be applied in order to maximize benefit for mother without harming the fetus.
\end{abstract}

Methods: We present a case of 37-year-old, five months pregnant woman who came to the hospital with chief complain of shortness of breath. Later on, she was found to have a stage IV Non-Small Cell Lung Cancer (NSCLC) with adenocarcinoma subtype.

Result: This may be the first lung cancer in pregnancy reported in Indonesia. Being a developing country, several challenges emerged before diagnosing patient of having lung cancer.

Conclusion: Careful considerations along with multidisciplinary approach are necessary to provide the best care for the patient.

Keywords: lung cancer, pregnancy, developing country

\section{INTRODUCTION}

Being inferior with low incidence in the beginning of the twentieth century, the incidence of lung cancer is swiftly increasing (1). In 2012, based on GLOBOCAN report, 1.8 million or $12.9 \%$ of all new cancer cases were lung cancer. Besides, over one million people died at the same year, which lead lung cancer to be on the first place as the most leading cause of cancer mortality (2).

Looking deeper into sexes, men were previously more prone to have lung cancer than women, but nowadays, the incidence of lung cancer in women continues to increase (3). On the contrary of its commonness, in some subgroup of women, especially in pregnant women, the cases of lung cancer are still rare (4). Cancer in pregnancy is found in one out of 1.000 pregnant women with breast, melanoma, cervical cancer and hematological malignancies as the most prevalent (5). In terms of lung cancer, based on report by Rivas et al, there had been 44 cases of pregnant women published until 2012 (6) Furthermore, Boussios et al stated that up to 2013, there had been 51 cases of lung cancer reported in English literature (7). Due to lack of cases reported and challenges that may be encountered especially between mother's and fetus' well-being, this report aims to give another perspective of diagnosing and treating lung cancer in expectant woman, especially in developing country.

\section{CASE PRESENTATION}

A 37-year-old, five months pregnant, Indonesian woman came to the hospital with chief complaint of increasing shortness of breath since two weeks before admission. Patient is a passive smoker (husband and parents are smokers) without history of other illnesses including cancer. Four months prior to admission, patient had a hemoptysis and was clinically diagnosed as tuberculosis. The reasons of being clinically diagnosed as tuberculosis were due to hemoptysis, young woman, non-smoker and malnutrition status. Bacteriology and radiology examination was not performed initially because there was no sputum 
produced and pregnancy status. Patient was then given anti-tuberculosis drug for two weeks prior to admission, she had shortness of breath, her doctor said that she had a fluid in both of her lung and pleurocentesis were performed with approximately 1 and 2 liter of fluid drawn from her lungs. However, after pleurocentesis, patient said that her symptoms returned and brought to Dr. Cipto Mangunkusumo Hospital $(\mathrm{CMH})$ for further evaluation. During admission, ultrasonography confirmed complex pleural effusion, then thoracocentesis followed by mini-drain installation were performed to drain the fluid. On physical examination, patient looked moderately ill, tachycardia 120 beats per minute (bpm), respiratory rate 26 times per minute, peripheral oxygen saturation $97 \%$ on 3 liter per minute nasal canulla. Moreover, right hemithorax had a muffled breath sound and hepatomegaly was found. On laboratory examination, hemoglobin 10.4, hematocrit $32.2 \%$, leukocyte $11,460 / \mathrm{mm} 3$, thrombocyte 297,000/mm3, erythrocyte sedimentation rate 60; Aspartate Aminotransferase (AST) and Alanine Aminotransferase (ALT) levels: 33 and $17 \mathrm{mg} / \mathrm{dL}$ respectively, total/direct/indirect bilirubin: 0.49/0.15/0.34 mg/dL, Ureum/Creatinine: 17/0.3 $\mathrm{mg} / \mathrm{dL}$. CT-scan was not conducted due to pregnancy.

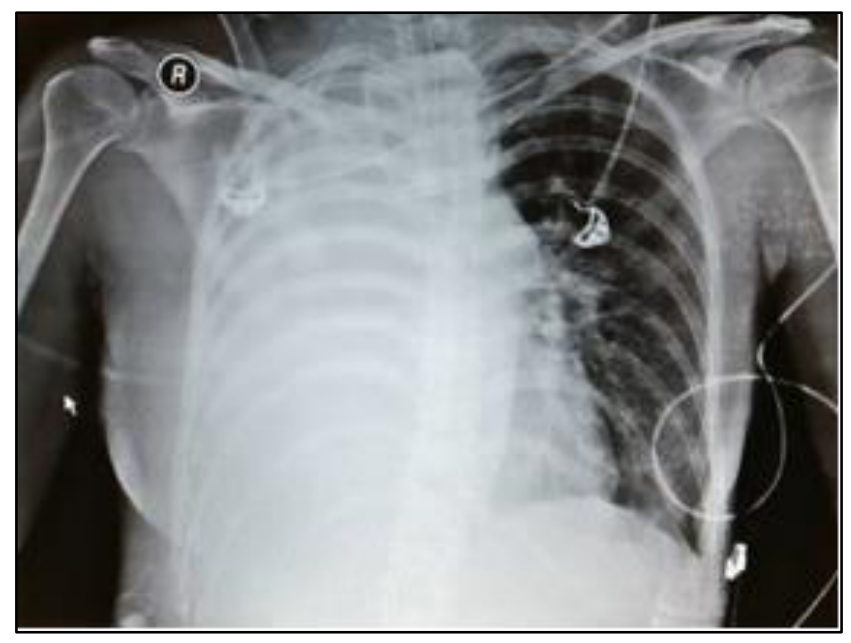

Figure 1. Post-bronchoscopy chest $x$-ray. Right pleural effusion, possibly with atelectasis. ETT with tip $4.3 \mathrm{~cm}$ above carina. No sign of pneumothorax, pneumomediastinum or subcutaneous emphysema.

Total stenosis of right superior trunk and partial stenosis of intermediate trunk due to compression and extralumen mass infiltration were found during bronchoscopy examination. Post bronchoscopy chest $x$ ray can be seen on figure 1 . From cytology examination of bronchoalveolar lavage and bronchial brushing showed a positive sign of poorly differentiated carcinoma. However, cytology from pleural fluid did not contain malignant tumor cell (figure 2). Lung biopsy showed non-small cell lung cancer (NSCLC) with adenocarcinoma subtype but no cancer cell was found in pleural biopsy. Due to the suspicion of having a mass in the liver, liver biopsy was performed. Liver metastasis with differential diagnosis of cholangiocarcinoma was found from the biopsy. Bronchial biopsy showed tumor cells with adenocarcinoma subtype (figure 3).

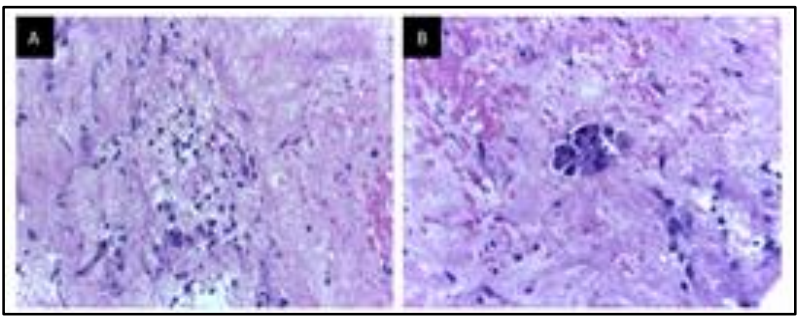

Figure 2. Pleura biopsy showed no malignant cell (Hematoxylin Eosin stain, 400X). (A) Connective tissue with acute and chronic inflammatory cells; (B) Atypical cells.

(Image courtesy: Department of Anatomic Pathology, Faculty of Medicine Universitas Indonesia-Dr Cipto Mangunkusumo National General Hospital)

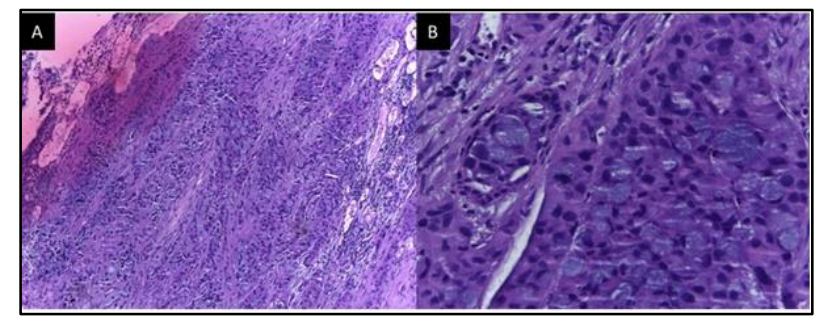

Figure 3. Bronchial biopsy. (A) Tumor tissue with solid, infiltrative, round-shape, eccentric, hyperchromatic, partly vesiculer, distinct nucleus, eosinophilic cytoplasm partly with mucinous and signel ring cell appearance; (B) Mitotic tumor cells.

(Image courtesy: Department of anatomic Pathology, Faculty of Medicine Universitas Indonesia-Dr Cipto Mangunkusumo National General Hospital)

Out of these examinations, lung cancer with liver metastasis and bilateral pleural effusion was confirmed (stage four lung cancer). Evaluation of fetomaternal consultant from Obstetrics and Gynecology Department showed 23 weeks pregnancy with normal fetal activity, no anatomical malformation, tendency of small fetus, no sign of hypoperfusion, funneling and shortening ( 7 $\mathrm{mm}$ ) cervix.

\section{DISCUSSION}

This case report may be the first lung cancer during pregnancy reported in Indonesia. On 2017 June 17th, authors tried to search with keywords "cancer and pregnancy and Indonesia" on Pubmed, but none was found. Moreover, until mid-year of 2017, there was no proper cancer registry which provides cancer-related information from residents across Indonesia, thus, there 
may be an underdiagnosed lung cancer in Indonesian pregnant women. To summarize, it can be said that lung cancer during pregnancy is a rare or underreported case.

Patient was initially presented with hemoptysis and was diagnosed with tuberculosis. Hemoptysis is a condition where blood expectorated from respiratory tract, either from bronchial or pulmonary vasculature $(8,9)$. Various etiology may contribute to the occurrence of this condition, starting from pulmonary tuberculosis (TB), bronchiectasis and necrotizing pneumonia in less developed countries, to lung cancer, pulmonary artery aneurysms and arteriovenous malformations in developed countries (9). Indonesia itself, being a developing country, is not only facing threats from infectious diseases, but also increasing number of noncommunicable diseases (10). However, because Indonesia has the world's second largest number of tuberculosis new cases in 2015 (11), pulmonary tuberculosis should be considered most likely as the cause of hemoptysis in this patient. Nevertheless, further evaluation indicated that this patient had lung cancer, which may cause hemoptysis as well. Being a double burden country, careful examination should be done in order to exclude either infection or lung cancer in patient with hemoptysis.

Diagnosing lung cancer in pregnancy remains a big issue due to its masking symptoms/signs, different cancer biology and behavior (affected by hormonal, immunosuppressive and environmental condition), rare cases, physicians' low level of suspicion, being reluctant to perform radiology examination, and late referral for invasive diagnostic procedure (7). In term of using radiology examination as one of the diagnostic modalities, fetal exposure is one of the main reasons behind the unwillingness. Based on guideline from The American College of Obstetricians and Gynecologists, radiation may be lethal to a fetus, but it depends on the dose and time of exposure. High-dose of radiation (more than $1 \mathrm{~Gy}$ ) during early embryogenesis may damage the process of development. However, the radiation doses of diagnostic imaging are not as high as 1 Gy (12). In research done by Patel et al, based on animal studies, studies on survivors of atomic bombings in Japan and specific groups who were exposed to radiation for medical reasons, the lowest estimated threshold dose of radiation which may harm fetal is approximately $50 \mathrm{mGy}$ during 0-2 weeks after conception. The larger gestational age of a fetus, the more possible he/she may survive from higher dose of radiation (13). In comparison, the fetal radiation doses from chest X-Ray and chest CT scan are approximately 0.0005-0.01 mGy and 0.01-0.66 mGy (depends on gestational age, maternal body habitus and certain acquisition parameters) (14). Nevertheless, there are no known congenital malformations or mental retardation risk in diagnostic imaging-related ionizing radiation in the exposed fetus, especially after first trimester (7). Thus, we can say that chest X-Ray and CT-scan are considered relatively safe to be used as diagnostic methods in pregnant women, though careful judgment should be done to minimize the unneeded exposure. This patient was presented with pleural effusion which can be diagnosed using ultrasonography and further non-radiation procedure will be able to confirm her stage IV NSCLC with adenocarcinoma subtype, so CTscan was not needed for diagnosis. However, in order to know the therapeutic/intervention response of the patient, CT-scan/chest $x$-ray prior to or post the initiation of intervention is still a necessary diagnostic modality.

Most cases of lung cancer during pregnancy was presented with advanced stage NSCLC with adenocarcinoma type. Nine cases from an international collaborative showed that all of the pregnant women presented in advanced stages also lead to dismal prognosis.7 NSCLC also become the most common histological type (80-85\%), followed by small cell lung cancer (SCLC; $10-15 \%$ ) and the rest are carcinoid of the lungs. Of all lung cancer cases, $90 \%$ were related to smoking, but in pregnant women, more than $40 \%$ cases occured without evidence of smoking $(4,15)$.

Upon establishing the diagnosis of lung cancer, careful consideration should be made before administrating drug. There are several treatment options for lung cancer, starting from surgery as the main treatment, chemotherapy, radiotherapy and targeted therapy such as tyrosine kinase inhibitors (TKIs). Choosing the best treatment option should be based on histopathology subtype and stage of the disease (16). In pregnant women, despite scarce research done in this specific population, ESMO Guidelines Working Group recommend to use combination of Carboplatin and Paclitaxel only in second and third trimester as the main treatment modality in NSCLC. Patient who came in the first trimester should be observed until second trimester or consider pregnancy termination whenever urgent treatment is required; third trimester patient should also think about pre-term delivery prior to drug administration (5). Chemotherapy may increase the potential of congenital malformation and fetal adverse effects by 10 to $20 \%$ especially if it is administered in the first trimester. However, administration after the first trimester will have no different risk compared to normal population (17). The risk of developing fetal malformation also depends on the type of chemotherapeutic agent, where antimetabolites and alkylating agents have the highest risk, while taxanes and platinum derivatives are considered as safe to be used in second and third trimester, although long-term effect is yet to be proven (4).

Eventually, before making the decision, multidisciplinary team from pulmonologists, intensivists, hepatologists, medical oncologists, pathologists, 
obstetric and gynecologists and radiologists sat together to discuss the best treatment option. In addition, patient and families were explained about the maternal benefit and fetal risks before administrating the chemotherapy. Multidisciplinary approach, added by families and patient involvement, should be taken into account to evaluate potential maternal benefit and fetal risks before drug administration (4).

\section{CONCLUSION}

This may be the first lung cancer in pregnancy reported in Indonesia. Being a developing country, emerging of lung cancer during pregnancy remains a big issue, with a limitation of data, high incidence of infectious disease, which may mask the clinical presentation of lung cancer, unwillingness to undergo radiology examination and late access to more advanced diagnostic procedure may lead to the late diagnosis of the disease. Moreover, careful consideration should be done before administering the drug in order to maximize maternal benefit and lower fetal risks. Last but not least, multidisciplinary approach is highly recommended to provide the best care for the patient.

\section{ACKNOWLEDGMENT}

We would like to thank Ms. Fetty, doctor Nadya, doctor Pandu, Division of Respirology and Critical IllnessDepartment of Internal Medicine, Department of Anatomic Pathology, Faculty of Medicine Universitas Indonesia, Dr. Cipto Mangunkusumo National General Hospital for their support in preparing this manuscript.

\section{CONFLICT OF INTEREST}

Hereby, the authors would like to declare no conflict of interest.

\section{REFERENCES}

1. Dubey AK, Gupta U, Jain S. Epidemiology of lung cancer and approaches for its prediction: A systematic review and analysis. Chin J Cancer. 2016; 35: 71.

2. International Agency for Research on Cancer. Lung cancer. World Health Organization [internet]. 2012; [cited 2017 June 2]. Available from: http://globocan.iarc.fr/Pages/fact_sheets_population.asp $x$

3. Barrera-Rodriguez R, Morales-Fuentes J. Lung cancer in women. Lung Cancer: Targets and Therapy. 2012; 3:7989.

4. Rothschild SI. Lung cancer in pregnancy-A forgotten disease entity. Journal of Thoracic Oncology. 2016; 11(9): 1376-8.

5. Peccatori FA, Azim Jr HA, Orecchia R, Hoekstra HJ, Pavlidis $\mathrm{N}$, Kesic $\mathrm{V}$, et al. Cancer, pregnancy and fertility: ESMO clinical practice guidelines for diagnosis, treatment and follow up. Ann Oncol. 2013; 24(supplement 6): v160-70.

6. Rivas G, Llinas N, Bonilla C, Rubiano J, Cuello J, Arango N. Use of erlotinib throughout pregnancy: A case-report of a patient with metastatic lung adenocarcinoma. Lung Cancer. 2012; 77: 469-72.

7. Boussios S, Han SN, Fruscio R, Halaska MJ, Ottevanger PB, Peccatori FA, et al. Lung cancer in pregnancy: Report of nine cases from an international collaborative study. Lung Cancer. 2013; 82: 499-505.

8. Ashraf O. Hemoptysis, a developing world perspective. BMC Pulm Med. 2006; 6:1.

9. Ogunrombi A, Onakpoya U, Adewole O, Adesunkanmi A. Challenges in the management of massive haemoptysis in a resource-scarce facility. Tropical Doctor. 2011; 41:8-10.

10. Rodriguez-Fernandez R, Ng N, Susilo D, Prawira J, Bangs MJ, Amiya RM. The double burden of disease among mining workers in Papua, Indonesia: at the crossroads between old and new health paradigms. BMC Publich Health. 2016; 16: 951.

11. World Health Organization. Global tuberculosis report 2016. [internet]. 2016; [cited 2017 June 18]. Available from:

http://www.who.int/tb/publications/global_report/gtbr2 016_main_text.pdf?ua=1

12. American College of Obstetricians and Gynecologists. Guideline for diagnostic imaging during pregnancy and lactation. Committee Opinion No. 656. Obstet Gynecol. 2016; 127: e75-80.

13. Patel SJ, Reede DL, Katz DS, Subramaniam R, Amorosa JK. Imaging the pregnant patient for nonobstetric conditions: Algorithms and radiation dose condiderations. Radio Graphics. 2007; 27: 1705-22.

14. Tremblay E, Therasse E, Thomassin-Naggara I, Trop I. Guidelines for use of medical imaging during pregnancy and lactation. Radio Graphics. 2012; 32: 897-911.

15. Mitrou S, Petrakis D, Fotopoulos G, Zarkavelis G, Pavlidis N. Lung cancer during pregnancy: A narrative review. Journal of Advanced Research. 2016; 7: 571-4.

16. Amin Z, Jayalie VF, Rajabto W. Recent management of patients with advanced epidermal growth factor receptor mutation non-small cell lung cancer: Role of afatinib and lesson learned for developing countries. Acta Med Indones-Indones J Intern Med. 2017; 49(1): 79-88.

17. Yates R, Zhang J. Lung cancer in pregnancy: An unusual case of complete response to chemotherapy. Cureus. 2015; 7(12): e440. 